\title{
Aqueous solutions of HEC and hmHEC: effects of molecular mass versus hydrophobic associations on hydrodynamic and thermodynamic parameters
}

\author{
Saumil Sudhir Vadodaria $(\mathbb{D} \cdot$ Robert John English
}

Received: 27 August 2015/Accepted: 6 January 2016/Published online: 19 January 2016

(C) The Author(s) 2016. This article is published with open access at Springerlink.com

\begin{abstract}
Aqueous solutions of amphiphilic polymers usually comprise of inter- and intramolecular associations of hydrophobic groups often leading to a formation of a rheologically significant reversible network at low concentrations that can be identified using techniques such as static light scattering and rheometry. However, in most studies published till date comparing water soluble polymers with their respective amphiphilic derivatives, it has been very difficult to distinguish between the effects of molecular mass versus hydrophobic associations on hydrodynamic (intrinsic viscosity $[\eta]$ ) and thermodynamic parameters (second virial coefficient $A_{2}$ ), owing to the differences between their degrees of polymerization. This study focuses on the dilute and semi-dilute solutions of hydroxyethyl cellulose (HEC) and its amphiphilic derivatives (hmHEC) of the same molecular mass, along with other samples having a different molecular mass using capillary viscometry, rheometry and static light scattering. The weight average molecular masses $\left(M_{W}\right)$ and their distributions for the nonassociative HEC were determined using size exclusion chromatography. Various empirical approaches developed by past authors to determine [ $\eta$ ] from dilute solution viscometry data have been discussed. hmHEC with a sufficiently high degree of hydrophobic
\end{abstract}

S. S. Vadodaria $(\bowtie) \cdot$ R. J. English

School of Engineering and Built Environment, Edinburgh

Napier University, Edinburgh, UK

e-mail: saumilvadodaria@gmail.com modification was found to be forming a rheologically significant network in dilute solutions at very low concentrations as opposed to the hmHEC with a much lower degree of hydrophobic modification which also enveloped the hydrophobic groups inside the supramolecular cluster as shown by their [ $\eta]$ and $A_{2}$. The ratio $A_{2} M_{W} /[\eta]$, which takes into account hydrodynamic as well as thermodynamic parameters, was observed to be less for associative polymers compared to that of the non-associative polymers.

Keywords Intrinsic viscosity $\cdot$ Second virial coefficient - Associative polymers - Amphiphilic polymers $\cdot$ Static light scattering $\cdot$ HEC $\cdot$ hmHEC

\section{Introduction}

Polysaccharides are the most abundant polymeric materials in nature and are essential components of biological systems. Owing to the renewable nature of the polysaccharide sources (i.e. plants and bacteria), the diversity of structures and the properties such as hydrogen-bond formation with water (Dumitriu 2005), polysaccharides and their derivatives have been a topic for research as solutions, gels and polysaccharide/protein mixtures in aqueous media and have often found their applications as rheology modifiers, emulsifiers and as a part of the drug delivery systems (Dumitriu 2001). 
Cellulose is a naturally occurring linear polymer of $1,4-\beta$-D-anhydroglucose repeating units and is the most abundant polysaccharide. Structurally, cellulose is a semi-flexible polymer in which the rotation along the glycosidic linkages is restricted (Martinez-Richa 2012). Cellulose ethers such as hydroxyethyl cellulose and carboxymethyl cellulose make an important subset of cellulose derivatives due to their properties such as solubility in water, chemical stability and nontoxicity (Klemm et al. 1998). Hydroxyethyl cellulose is a cellulose ether which is essentially non-ionic in nature and is widely used as a rheology modifier in latex paints, protective colloid, binder and film former (Whistler and BeMiller 1993).

Hydrophobically modified hydroxyethyl cellulose (hmHEC) has been studied extensively over last few decades after its first academic investigation published by Landoll 1982 (Maestro et al. 2002b; Chassenieux et al. 2010; Zhang 2001; Laschet et al. 2004; Zhao and Chen 2007; Gonzalez et al. 2005; Patruyo et al. 2002). This polymer is synthesized by grafting hydrophobic 'sticker' groups across the hydrophilic backbone of HEC. The intra- and intermolecular aggregation of these hydrophobic groups results into the energy gain. The number of chains in an intermolecular aggregate is determined by the balance of the steric repulsion between the hydrophilic backbones in the vicinity of each other versus the energy gain due to the hydrophobic association. These intra- and intermolecular associations cause the solution viscosity to enhance following the formation of a reversible three-dimensional supramolecular network. One of the advantages of hmHEC over HEC is that the former can be an equally effective thickener at relatively lower molecular mass compared to the latter as a result of the supramolecular network. Lower molecular mass reduces the elastic effects within the fluid, which could reduce the spattering in paints applied using rollcoating (Davison and Lane 2003). Moreover, the hydrophobic associations can be broken by extensional strain, lowering the extensional viscosity which is recovered gradually after the deformation stops (Goodwin and Hughes 2008).

The intramolecular hydrophobic associations present in the hmHEC coils at a dilute concentration cause the coils to collapse which reduces the hydrodynamic volume per coil and hence the intrinsic viscosity compared to those of an unmodified HEC with similar degree of polymerization. However, since these chains often also form supramolecular clusters/ aggregates, the intrinsic viscosity no longer necessarily represents the average hydrodynamic volume of a coil made of a single polymer chain, but that of a coil of an aggregate (Maestro et al. 2002a; Simon et al. 2003; Laschet et al. 2004). Hence an accurate determination of intrinsic viscosity leads to a better understanding of the nature of intra- and intermolecular associations.

The determination of intrinsic viscosity also presents a challenge in the case of hmHEC solutions. At a concentration below the critical overlap concentration $c^{*}$ (the way it is understood for a non-associative polymer with the same degree of polymerization), the formation of a rheologically significant supramolecular network often results in deviation from the viscometric behavior as predicted by the well known Huggins equation (Landoll 1982). This is a common phenomenon among many associative polymers, such as the hydrophobic derivatives of both carboxymethyl cellulose (Charpentier-Valenza et al. 2005) and carboxymethyl pullulans (Simon et al. 2003), and the hmHEC (Maestro et al. 2002a; Landoll 1982). As an alternative approach, many different empirical methods (i.e. Martin, Schulz-Blaschke, Fedors, Fuoss and Heller) have been used to calculate the intrinsic viscosity of associative derivatives of polymers such as dextran (Rotureau et al. 2006; Durand 2007b), polyacrylamide (Maia et al. 2011; Ma et al. 2003) and cationic polyelectrolytes (Dragan and Ghimici 2001). In most cases, the use of alternative empirical methods (especially Fedors equation) has not only provided a more accurate prediction of the viscometric behavior at a much wider concentration range compared to that of Huggins, but the knowledge of the empirical parameters obtained from these relationships has been helpful for interpreting the nature of the polymersolvent interaction. However, these empirical methods have never been reported to be used for determining the intrinsic viscosity of hmHEC.

These extrapolation methods have been widely used in the past 20 years to characterize the solutions of many biopolymers and synthetic polymers. Thus they have been employed in the present study and the intrinsic viscosity values obtained from them are compared. Also, $[\eta]$ has been interpreted to understand the nature of intra- and intermolecular associations in the hmHEC polymers. Numerous studies have reported the reduction of intrinsic viscosity in 
associative polymers as a result of intramolecular associations, e.g. hydrophobic derivatives of polymer such as dextran (Rotureau et al. 2006; Durand 2007a, b), carboxymethyl cellulose (Charpentier-Valenza et al. 2005), polyacrylamide (Maia et al. 2011) and HEC (Maestro et al. 2002a). The degree of hydrophobic modification has been sufficiently high in all the above studies to cause the polymer coil to have a lower size compared to the coil of its corresponding nonassociative derivative with similar molecular mass (due to a denser packing of chains within a coil) even though the coil was likely to be an aggregate of more than one polymer chains.

In many of the studies regarding associative polymers mentioned above, the second virial coefficient $\left(A_{2}\right)$ values of the associative polymers have been reported to be lower compared to their corresponding non-associative polymers, indicating a relatively inferior solvent quality in terms of polymersolvent interaction owing to hydrophobic interactions. While a relatively lower degree of hydrophobic modification still allows intra- and intermolecular associations to occur, the hydrophilic polymer backbone may be capable to envelope the hydrophobic groups almost completely, hence preventing the formation of a supramolecular network at the concentrations lower than the $c^{*}$. However, there are no published studies which discuss about the intramolecular and intermolecular associations in dilute solutions of polysaccharides with a degree of hydrophobic modification which is not high enough to cause the formation of a supramolecular network according to the best of our knowledge.

The self-assembly of hmHEC cannot be studied entirely on the basis of intrinsic viscosity determination, as the value of $[\eta]$ alone cannot be interpreted as an evidence of intra- or intermolecular association. Nor the value of $A_{2}$ alone can be used for this purpose, as the associative polymers do not always have negative $A_{2}$ but still form aggregates. In order to reconcile [ $\eta$ ] (a measurement of hydrodynamic volume of the coil) with the second virial coefficient $A_{2}$ (a measurement of thermodynamic quality of solventpolymer interaction), an empirical relationship $[\eta]=A_{2} M_{W}$ has been used by many previous authors (Martins et al. 2006; Lee 1992; Yamakawa 2001; Teraoka 2002). As $A_{2} M_{W}=0$ under theta conditions, this equation requires $A_{2}$ to be positive and sufficiently high (the previous authors mentioned above have used values of $A_{2} \geq 5.21 \times 10^{-6} \mathrm{~mol} \mathrm{dm} \mathrm{g}^{-2}$ for

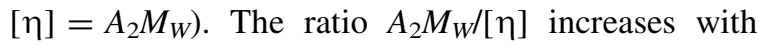
the thermodynamic solvent quality. In the present study, an attempt has been made to correlate light scattering with $[\eta]$ for associative cellulosic polymers for the first time.

In the present study, hmHEC polymers with both high and low degrees of hydrophobic modifications have been characterized. For the convenience of analysis, they have been divided in two sets. Set A consists of a non-associative HEC and an hmHEC with relatively high degree of hydrophobic modification. Both the polymers have different molecular masses. Another set (set B) contains three polymers of similar molecular masses, one non-associative HEC (referred to as hmHEC-Blank) and two hmHECs (referred to as hmHEC-1 and hmHEC-2) with expectedly different degrees of hydrophobic modification which are lower compared to that of the hmHEC in set A. The differences between the dilute solution behaviour of hmHECs with high and low degrees of hydrophobic modification (specifically, the polymerpolymer and polymer-solvent interactions) are discussed following the characterization.

\section{Materials and methods}

\section{Materials}

Hydroxyethyl cellulose (HEC) was obtained from Aqualon Hercules under the brand name Natrosol HEC $250 \mathrm{MR}$, with an average molecular substitution of 2.5 ethylene oxide units per anhydroglucose repeating unit. The weight average molecular mass $M_{W}$ was measured to be about $7.20 \times 10^{5} \pm$ $0.3 \%$ Da through aqueous size exclusion chromatography with $0.1 \mathrm{M} \mathrm{NaNO}_{3}+10^{-3} \mathrm{M} \mathrm{NaN}$ using multi-angle laser light scattering. The solution was filtered through Milipore $0.45 \mu \mathrm{m}$ filter. The flow rate of the mobile phase was $0.5 \mathrm{ml} / \mathrm{min}$ through Suprema mixed bed column containing nearly monodisperse beads of polyhydroxymethacrylate copolymer. Results were treated using Zimm method. A manually measured value of the refractive index increment $(\mathrm{d} n /$ $\mathrm{d} c=0.107)$ using Optilab DSP differential refractometer was used in the calculation.

Hydrophobically modified hydroxyethyl cellulose (hmHEC) was obtained from Aqualon Hercules as 
Natrosol Plus 330 CS. Aqueous size exclusion chromatography could not be used for hmHEC as a result of supramolecular aggregation (Islam et al. 2000). The molecular mass according to literature (Gonzalez et al. $2005)$ is $M_{W}=3.0 \times 10^{5} \mathrm{Da}$, with $M S=2.5$ ethylene oxide groups per sugar unit, while hydrophobic pendant groups have been reported as hexadecyl chains with approximately 0.01 molar substitution (Gonzalez et al. 2005).

\section{Synthesis}

Three different 'versions' of hmHEC were synthesized using phase transfer catalyst in a heterogeneous reaction (Swift et al. 1997). HEC 250 MR was used as the substrate. All three reactions were identical in all other aspects except for stoichiometry of hydrophobic modifier.

$52.25 \mathrm{~g}$ of Natrosol HEC $250 \mathrm{MR}+340 \mathrm{ml}$ of 70:30 ethanol:toluene $+32 \mathrm{ml}$ of deionized water was taken. A three necked round bottom flask was fitted with a PTFE stirrer-motor, nitrogen inlet, serum cap with a mercury thermometer and a Friedrich condenser. The mixture was stirred for $1.5 \mathrm{~h}$ during which $\mathrm{N}_{2}$ was continuously purged. Then the mixture was treated drop wise with $40 \mathrm{~g}$ of $40 \% \mathrm{w} / \mathrm{w}$ aqueous tetrabutylammonium hydroxide over $10 \mathrm{~min}$ through a dropping funnel. The mixture was then stirred for $1 \mathrm{~h}$ under $\mathrm{N}_{2}$. After this step, no hydrophobe was added in case of hmHEC-blank whereas 3.5 and $7.0 \mathrm{~g}$ of 1,2epoxyoctadecane were added drop wise respectively for hmHEC-1 and hmHEC-2.

This mixture was heated under reflux under $\mathrm{N}_{2}$ for $3 \mathrm{~h}$. It was then cooled to room temperature and neutralized to $\mathrm{pH}=4$ by adding glacial acetic acid in an ice bath, followed by $15 \mathrm{~min}$ of stirring. The solid precipitates of polymer were then collected by vacuum filtration, washed in a blender four times with $200 \mathrm{ml}$ of 50:50 mixture of acetone:water each time and twice with $200 \mathrm{ml}$ of pure acetone each time. The solid powder was then dried under vacuum overnight with a yield of 47.5, 46 and $46.8 \mathrm{~g}$ of hmHEC-blank, hmHEC-1 and hmHEC-2 respectively. The molecular mass was determined for the non-associative polymer hmHEC-Blank using size exclusion chromatography coupled with multi-angle laser light scattering following the same procedure described above. Due to very low degree of hydrophobic modification, the results of FTIR and Carbon-Oxygen ratio analysis using EDX (energy-dispersive X-ray spectroscopy) failed to provide a quantitative estimation of molecular substitution of hydrophobic groups. All three samples from set $\mathrm{B}$ were also tested using ${ }^{13} \mathrm{C} \mathrm{NMR}$, which also failed to quantify the hydrophobic modification, possibly due to apparently very low molecular substitution as well as relatively less natural abundance of ${ }^{13} \mathrm{C}$ isotope among carbon atoms.

\section{Solutions}

All solutions were prepared in $0.1 \mathrm{M} \mathrm{NaNO}_{3}+10^{-3}$ $\mathrm{M} \mathrm{NaN}$ in water by mixing with solid powder of respective polymers. The bottles containing mixtures were placed on a roller mixer for up to $24 \mathrm{~h}$. All solutions were characterized within $48 \mathrm{~h}$ after the mixing.

\section{Characterization}

\section{Dilute solution viscometry}

Stock solutions of HEC and hmHEC (set A and B) were prepared at $0.1-0.2 \mathrm{~g} \mathrm{dl}^{-1}$ concentration in the solvent $\left(0.1 \mathrm{M} \mathrm{NaNO}_{3}+10^{-3} \mathrm{M} \mathrm{NaN}_{3}\right.$ in water). Flow times of the solvent, stock solution and its dilutions (with $2 \mathrm{ml}$ of solvent each time) were measured using an Ubbelohde viscometer (Cannon Instrument Company, 75 J349 series) immersed in a water bath at $25 \pm 0.1{ }^{\circ} \mathrm{C}$. The measurements were repeated three times and the average flow time was calculated for each concentration. Relative viscosity was derived by dividing the average solution flow time with the average solvent flow time. The measurements of average flow time were continued till the relative viscosity was below 1.2 .

\section{Steady shear rheometry}

AR-2000 controlled stress rheometer (TA Instruments) fitted with a concentric cylinder (Stator inner radius $15 \mathrm{~mm}$, rotor outer radius $14,42 \mathrm{~mm}$ of cylinder immersed) was used to measure the shear rate dependent viscosity. The sample was subjected to shear-conditioning for $180 \mathrm{~s}$ at $3 \mathrm{~s}^{-1}$ shear rate followed by $180 \mathrm{~s}$ of equilibrium time. The range of applied shear rate was from 0.01 to $1000 \mathrm{~s}^{-1}$ with 30 data points (stepped rate profile) logarithmically 
equidistant from one another. Each shear rate was applied for $45 \mathrm{~s}$ and the average shear stress during last $15 \mathrm{~s}$ was measured. The reproducibility of all experiments was verified by repeating them. For some samples, the shear rate sweeps were repeated with longer time for each shear rate to confirm the steady state.

\section{Theory}

Intrinsic viscosity

A solution is said to be dilute and ideal if the average distance between the solute molecules is large enough to neglect the intermolecular interactions. For an ideal and dilute solution of hard spheres, Einstein proposed the following equation (Einstein 1920):

$\eta_{s p}=2.5 \frac{N_{A} n V_{n}}{V}$

$N_{A}$ is the Avogadro number, $n$ is the mole of particles present, $V$ is the total volume of solutions in mol dm $\mathrm{dm}^{-1}$ and $V_{n}$ is the hydrodynamic volume occupied by each sphere. $\eta_{s p}$ (specific viscosity) is $\left[\left(\eta / \eta_{s}\right)-1\right]$ or $\left[\eta_{\text {rel }}-1\right]$ where $\eta, \eta_{s}$ and $\eta_{\text {rel }}$ are solution viscosity, solvent viscosity and relative viscosity respectively.

In Eq. (1), $n / V$ can also be expressed using the concentration $c(\mathrm{w} / \mathrm{v})$ as given below:

$n=c \frac{V}{M_{W}}$

Substituting the value of $n$ in the Eq. (1) and rearranging:

$\frac{\eta_{s p}}{c}=[\eta]=2.5 \frac{N_{A} V_{n}}{M_{W}}$

It can be seen that according to the above equation, the intrinsic viscosity $[\eta]$ is also referred to as the reduced viscosity $\eta_{s p} / c$ which is independent of concentration, as opposed to the limiting value of reduced viscosity at infinite dilution. However, this expression is held true only for ideal solutions with no interaction between the spherical coils (Kulicke and Clasen 2004).

For real solutions, the concentration has to be vanishingly small for their behavior to be close to an ideal solution, i.e. have negligible interaction between the spherical coils due to larger distances (Kulicke and Clasen 2004). $\lim _{c \rightarrow 0} \frac{\eta_{s p}}{c}=[\eta]_{\theta}=2.5 \frac{N_{A} V_{n}}{M_{W}}$

In the above equation, $[\eta]_{\theta}$ describes the intrinsic viscosity of a polymer coil with unperturbed dimensions in an ideal solution, i.e. in a theta solvent. Again, for ideal solutions the intrinsic viscosity can further be described simply by rearranging the Eq. (4). The volume fraction of polymer coils $\varphi$ is defined as the ratio of the combined volume of the coils to the total solution volume.

$[\eta]_{\theta} c=2.5 N_{A} n \frac{V_{n}}{V}=2.5 N_{A} n \phi$

Overlap concentration $\left(c^{*}\right)$

The polymer coils are spatially separated from one another in dilute solutions. However, when the polymer concentration is increased, the average distance between the coils is reduced until they eventually overlap and interpenetrate. The concentration associated with incipient interpenetration, at which all chains are in contact with their immediate neighbors and the density of chain segments across the system is invariant is termed the critical overlap concentration, $c^{*}$. This concentration (overlap concentration $c^{*}$ ) is of prime importance because the mechanism of viscosification is changed at concentrations greater than $c^{*}$. For $c<c^{*}$ the curvature of the flow lines around the individual polymer coils is responsible for the increase in viscosity, as opposed to $c>c^{*}$ when the network composed of overlapping coils resists the flow.

The overlap concentration $c^{*}$ can be determined using intrinsic viscosity measurement. It is apparent that intrinsic viscosity does not represent actual viscosity but rather is a measure of the hydrodynamic volume of the coil of uncharged polymer chains (Dumitriu 2005). Greater hydrodynamic volume of chains per unit mass of polymer would result in less concentration of polymer required in order to occupy the entire volume of the solution by the coils.

The determination of $c^{*}$ for a polymer-solvent system can thus be performed by assuming an inverse proportionality of $c^{*}$ to intrinsic viscosity (i.e. $c^{*}=k /$

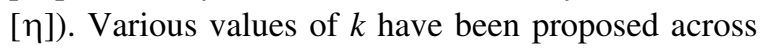
the literature-for example $k=0.77$ (Graessley 1980), $k=1$ (Teraoka 2002) and $k=2.5$ (Kulicke and Clasen 2004). The value $k=0.77$ is based on the 
assumption that the molecular coils behave as monodisperse spheres and their corresponding maximum packing fraction corresponds to the overlap concentration $c^{*}$, while the situation $k=1$ is premised on the solvated polymer coils occupying the entire solution volume.

\section{Second virial coefficient $\left(A_{2}\right)$}

Van't Hoff proposed the following equation for osmotic pressure $\Pi$ of a dilute and ideal polymer solution (Kamide and Dobashi 2000; van't Hoff 1903). To observe this equation is one of the characteristics of an ideal solution (Teraoka 2002).

$$
\frac{\Pi}{R T}=\frac{c}{M_{W}}
$$

here $R$ is the gas constant and $T$ is the absolute temperature. The right hand side of this equation undergoes a virial expansion for non-ideal solutions (Teraoka 2002).

$\frac{\Pi}{R T}=\frac{c}{M_{W}}+A_{2} c^{2}+A_{3} c^{3}+\cdots$

$A_{2}$ and $A_{3}$ are second and third virial coefficients respectively. It can be seen that the magnitude of $A_{2}$ indicates how much deviation from the ideal nature has occurred. $A_{2}=0$ indicates an ideal solution. Positive and negative $A_{2}$ describe 'good' and 'bad' solvent quality respectively. We see that Eq. (7) for non-ideal solutions can be transformed into the following at very low concentration when higher order terms are ignored:

$\frac{\Pi \mathrm{M}_{\mathrm{W}}}{c R T}=1$

The same equation at $c=c^{*}$ and a positive high value of $A_{2}$ (Indicating 'good' solvent quality) can be expressed as:

$\frac{\Pi \mathrm{M}_{\mathrm{W}}}{c^{*} R T}=1+A_{2} M_{W} c^{*} \approx A_{2} M_{W} c^{*}$

The magnitude of $A_{2} M_{W}$ is an indicator of how much the solution deviates from the ideal behavior (Teraoka 2002). Dividing Eq. (8) with Eq. (9),

$A_{2} M_{W} c^{*} \approx \frac{c}{c^{*}}$

At $c=c^{*}$,
(Teraoka 2002) $A_{2} M_{W} \approx \frac{1}{c^{*}} \approx[\eta]$

The above relationship has been utilized to determine $c^{*}$ of hydroxypropyl cellulose (Martins et al. 2006). The dimensionless ratio of $A_{2} M_{W} /[\eta]=0$ (which indicates theta conditions) corresponds to $[\eta] /[\eta]_{\theta}=1$ which increases with $A_{2} M_{W} /[\eta]$ (Lee 1992).

For flexible chains within good solvent, $A_{2} M_{W} /[\eta]$ ranges between 1.0 and 1.2 (Yamakawa 2001). Thus it can be argued that while $[\eta]_{\theta}$ increases with the molecular mass, [ $\eta$ ] of flexible chains in 'good' solvent increases with $A_{2} M_{W}$. It should be noted here that the second virial coefficient could also be determined from static light scattering, through the following expression usually attributed to Debye:

$\frac{K C}{R_{\theta}}=\frac{1}{M_{W}}+2 A_{2} c$

In the above expression, $K$ is an optical constant and $R_{\theta}$ is the excess Rayleigh ratio.

$K=\frac{2 \pi^{2} n_{0}^{2}}{N_{A} \lambda_{0}^{4}}\left(\frac{d n}{d c}\right)^{2}$

here, $n_{0}$ is the solvent refractive index, $\lambda_{0}$ is the wavelength of light and $\mathrm{d} n / \mathrm{d} c$ is the refractive index increment for the polymer-solvent system, which is measured independently via differential refractometry.

The following expression defines the excess Rayleigh ratio:

$R_{\theta}=\frac{\left(I_{\theta}-I_{\theta, \text { solvent }}\right) r^{2}}{I_{0} V}=f \frac{E_{\theta}-E_{\theta, \text { solvent }}}{E_{\text {laser }}}$

$I_{\theta}$ and $I_{\theta \text {, solvent }}$ are the scattered light intensity of the solution and the solvent respectively. $V$ is the solution volume and $r$ is the distance between the detector and the scattering volume. Instrumentally, however, $R_{\theta}$ is determined by $E_{\theta}, E_{\theta}$, solvent and $E_{\text {laser }}$, the detector signal voltages of the solution, solvent and laser respectively (Podzimek 2011; Kamide and Dobashi 2000).

Aqueous solutions of associative polymers

Graft polymers such as hmHEC with hydrophobic side chains and hydrophilic backbone are a subset of associative polymers. In dilute aqueous solutions of 
such polymers, the interactions (intra- and intermolecular) between these hydrophobic groups lead to the formation of physical bonds which are reversible during the experimental time scale.

The formation of aggregates is controlled by two essential factors: the loss of configurational entropy of the individual chains and the energy gain due to the association of the hydrophobic segments. The association is thermodynamically stable if the energy gain exceeds the entropic penalty (Rubinstein and Dobrynin 1997). As a result of these associations, the polymer chains undergo partial collapse as well as aggregation in a polydisperse array of supramolecular clusters/ micelles (English et al. 2002). It has been reported that the micelles exist in aqueous solutions of hmHEC even at very dilute concentration $\left(c<10^{-4} \mathrm{~g} \mathrm{dl}^{-1}\right)$ (Maestro et al. 2002a).

The solution viscosity is very close to the solvent viscosity at very low concentrations of associative polymers with the presence of small clusters. The relaxation time of these clusters is shorter than their lifetime. Hence the rheology of associative polymer solutions in this concentration regime is similar to that of the systems formed by permanent bonds because the associations do not undergo frequent cleavage.

At higher concentrations, larger and polydisperse clusters emerge. These clusters undergo a continuous process of breaking and reforming by dissociation and association of the hydrophobic groups (English et al. 2002). At this stage, the overall number of intermolecular interactions increases. A further increase in concentration leads to the formation of a threedimensional reversible network spanning across the solution volume and this network becomes responsible for the rheological behavior of the solution as opposed to the polydisperse clusters which governed the flow properties at lower concentrations. In non-associative polymers, a sudden change of rheological behavior occurs usually at the coil overlap concentration $\left(c^{*}\right)$. However, due to the presence of the reversible network of clusters, $c^{*}$ is less relevant for associative polymer solutions (Abdala et al. 2004; Simon et al. 2003).

Owing to the reasons discussed above, the intrinsic viscosity value for amphiphilic polymer solutions does not truly represent the hydrodynamic volume of a coil of a single chain but merely that of an average coil with both intra- and intermolecular associations (Rotureau et al. 2006). However, the [ $\eta]$ and $A_{2}$ values of these polymer solutions could be compared with those of their corresponding non-associative polymer solutions to study the supramolecular network.

Empirical methods for intrinsic viscosity determination

Considering the relationship of intrinsic viscosity with the chain dimensions and solvent quality, its accurate determination is essential. There are several empirical relationships available in order to calculate the intrinsic viscosity using viscosity measurement data:

$\frac{\eta_{s p}}{c}=[\eta]+K_{H}[\eta]^{2} c \quad$ (Huggins 1942)

$\frac{\ln \eta_{r}}{c}=[\eta]-\left(K_{H}-\frac{1}{2}\right)[\eta]^{2} c \quad($ Kraemer 1938)

$\frac{\eta_{s p}}{c}=[\eta] \exp \left(K_{M}[\eta] c\right) \quad$ (Bungenberg de Jong etal. 1932) Unpublished works of A F Martin;

$\frac{\eta_{s p}}{c}=[\eta]+[\eta] K_{S B} \eta_{s p} \quad($ Schulz and Blaschke 1941)

$\frac{1}{2}\left(\frac{c}{\eta_{s p}}+\frac{c}{\ln \eta_{r e l}}\right)=\frac{1}{[\eta]}-\left(\frac{k_{1}-k_{2}}{2}\right) c$

(Heller 1954)

$$
\begin{aligned}
& \frac{c}{\eta_{s p}}=A \sqrt{c}+\frac{1}{[\eta]} \quad(\text { Fuoss and Strauss 1948) } \\
& \frac{1}{2\left(\eta_{\text {rel }}^{1 / 2}-1\right)}=\frac{1}{[\eta] c}-\frac{1}{[\eta] c_{\max }} \quad \text { (Fedors 1979) }
\end{aligned}
$$

Arguably among the equations illustrated above, Fedors (1979) equation has been reported to be applicable in describing the viscometric data for solutions of associative (hydrophobically modified) and non-associative water soluble polymers and polyelectrolytes. Among all the extrapolation methods describe above, the Fedors equation has been reported within the past few years to be applicable for many polymers and to a much wider range of concentration with good linearity compared to other equations 
(Rotureau et al. 2006; Maia et al. 2011; Pavlov et al. 2006; Durand 2007b).

The concentration parameter $c_{\max }$ has the dimensions of concentration and has been claimed by Fedors (1979) to indicate the upper limit of concentration that can be described by this equation, i.e. $c<c_{\max }$.

Interestingly, Durand (2007a) noted that the left side terms of both the Heller and Fedors equations are in close agreement up to $\eta_{s p}=3$. Based on this premise the following relationship was proposed (Durand 2007a):

$c_{\max }[\eta]=\frac{1}{K_{H e}}$

\section{Results and discussion}

\section{Calculation of intrinsic viscosity}

The viscometric data corresponding to all the extrapolation relationships that give $[\eta]$ as the $y$-intercept of the straight line (i.e. Huggins, Kraemer, Martin and Schulz-Blaschke), has been plotted in Figs. 1 and 2. These data for HEC and hmHEC solutions (set A) have been presented in Fig. 1a, b and those for hmHEC-Blank, hmHEC-1 and hmHEC-2 (set B) solutions have been plotted in Fig. 2a-c. The intrinsic viscosity values for all polymers have been given in Table 1. There is a good agreement between these three empirical models for the intrinsic viscosity values for all polymers.

In Fig. 1a, the onset of non-linearity from Huggins equation starts at a concentration which is lower than the overlap concentration defined by $1 /[\eta]$ and closer to $0.77 /[\eta]$. In Fig. $1 b$, the deviation from Huggins equation which occurs at the concentration below $0.77 /[\eta]$ can be attributed to the reversible supramolecular network present in hmHEC solutions. This behavior does not indicate a classical overlap but the presence of a rheologically significant network across the system. This phenomenon has been reported in carboxymethyl cellulose (Charpentier-Valenza et al. 2005), carboxymethyl pullulans (Simon et al. 2003) and hmHEC (Maestro et al. 2002a; Landoll 1982). As explained in the theory section, these studies suggest that the aggregates of more than one polymer chain exist even at very low concentrations of amphiphilic polymers.

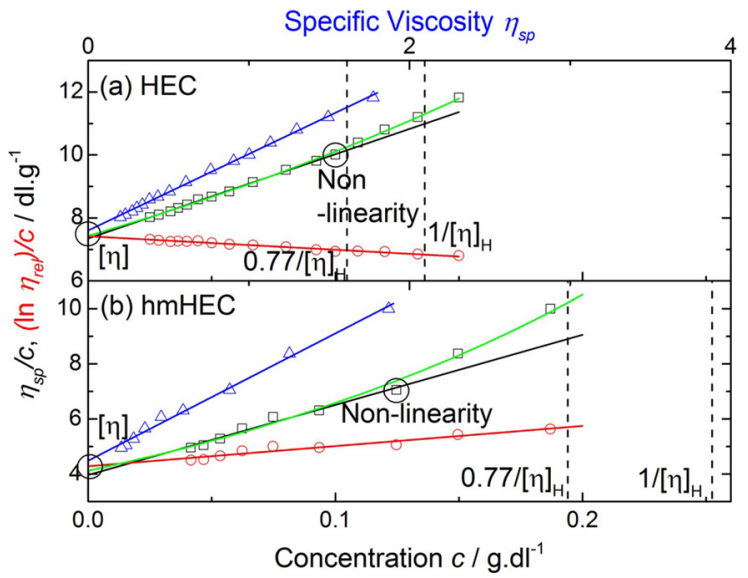

Fig. 1 Reduced viscosity values $\left(\eta_{s p} / c\right)$ for a HEC and b hmHEC (set A) plotted against concentration $c$ (open square) and against specific viscosity $\eta_{s p}$ (open triangle). Inherent viscosity values $\left(\ln \eta_{\text {rel }} / c\right)$ plotted against concentration $c$ (open circle). Black, red, green and blue solid lines are fits for Huggins, Kraemer, Martin and Schulz-Blaschke equations respectively. Two different values of critical overlap concentration have been indicated corresponding to $1 /[\eta]$ and $0.77 /[\eta]$ respectively. (Color figure online)

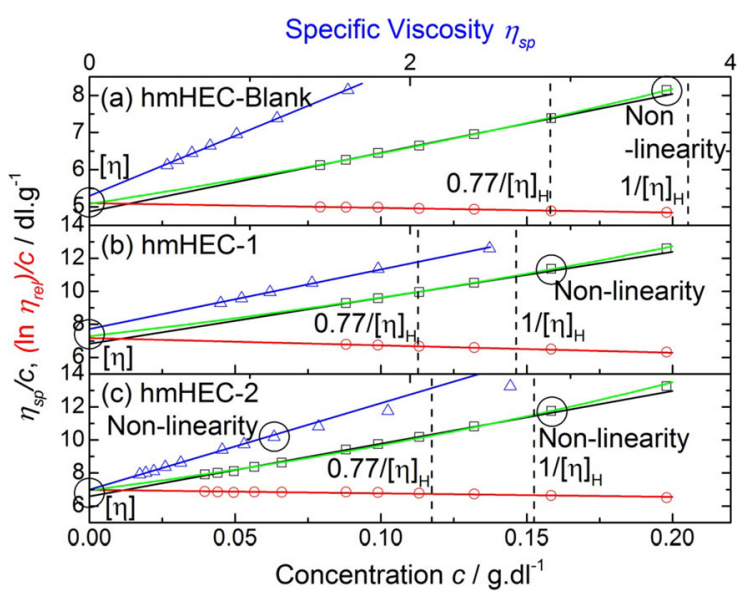

Fig. 2 Reduced viscosity values $\left(\eta_{s p} / c\right)$ for a hmHEC-Blank, b hmHEC-1 and c hmHEC-2 (set B) plotted against concentration $c$ (open square) and against specific viscosity $\eta_{s p}$ (open triangle). Inherent viscosity values $\left(\ln \eta_{\text {rel }} / c\right)$ plotted against concentration $c$ (open circle). Black, red, green and blue solid lines are fits for Huggins, Kraemer, Martin and Schulz-Blaschke equations respectively. Two different values of critical overlap concentration have been indicated corresponding to $1 /[\eta]$ and $0.77 /[\eta]$ respectively. (Color figure online)

The viscometric data in Fig. 1a, b is in a better agreement with Kraemer equation. However, in Fig. $1 \mathrm{~b}$ the slope of the linear fit to the data corresponding to Kraemer equation is positive, which 
Table 1 The intrinsic viscosity values $\left(\mathrm{dl} \mathrm{g}^{-1}\right)$ and the empirical constants obtained from Huggins, Kraemer and Schulz-Blaschke equations for all set $\mathrm{A}$ and B polymers and the concentration $c_{N L}$ at which the corresponding data exhibits a deviation from Huggins equation

\begin{tabular}{lllllllllll}
\hline Polymer & {$[\eta]_{\mathrm{H}}$} & $K_{H}$ & {$[\eta]_{\mathrm{K}}$} & \multicolumn{1}{c}{$K_{K}$} & $K_{H}-K_{K}$ & {$[\eta]_{\mathrm{SB}}$} & $K_{S B}$ & {$[\eta]_{\mathrm{M}}$} & $K_{M}$ & $c_{N L}$ \\
\hline HEC & 7.34 & 0.497 & 7.41 & -0.0806 & 0.578 & 7.60 & 0.320 & 7.43 & 0.414 & 0.100 \\
hmHEC & 3.96 & 1.620 & 4.28 & 0.4000 & 1.220 & 4.49 & 0.669 & 4.12 & 1.140 & 0.130 \\
hmHEC-Blank & 4.87 & 0.667 & 5.10 & -0.0497 & 0.717 & 5.30 & 0.336 & 5.08 & 0.467 & 0.200 \\
hmHEC-1 & 6.83 & 0.596 & 7.18 & -0.0866 & 0.683 & 7.72 & 0.257 & 7.28 & 0.384 & 0.160 \\
hmHEC-2 & 6.56 & 0.739 & 6.92 & -0.0264 & 0.765 & 6.99 & 0.412 & 6.95 & 0.477 & 0.160 \\
\hline
\end{tabular}

has also been observed for hydrophobically modified methacrylic acid-ethyl acrylate copolymers (Lau et al. 2002). Also, the exponential fit corresponding to the Martin equation on the same set of data agrees with the data points deviating from linearity as defined by the Huggins equation. The linear fit on the plot of reduced viscosity against specific viscosity (corresponding to Schulz-Blaschke equation) is also in a good agreement with the data points.

In Fig. 2a-c for the set $\mathrm{B}$ polymers the transitions from dilute to non-dilute regime shown by the onset of non-linearity from Huggins equation are very close to the respective concentrations depicted by $1 /[\eta]$, including for the associative polymers hmHEC-1 and hmHEC-2. This behavior is in contrast to that of the hmHEC of set A. This can be explained by the difference in the degree of hydrophobic substitution. Since the degree of hydrophobic modification for both hmHEC-1 and hmHEC-2 in set A is much lower than that of the hmHEC in set $\mathrm{B}$, the formation of supramolecular network with reversible hydrophobic associations is likely to occur at much higher concentrations. The hmHEC in set $\mathrm{A}$ has about 0.01 molar substitution of C-16 alkyl chains (Gonzalez et al. 2005). The set B amphiphilic polymers hmHEC-1 and hmHEC-2 have much lower degree of modification of $\mathrm{C}-18$ chains. The viscometric data in Fig. $2 \mathrm{a}, \mathrm{b}, \mathrm{c}$ is also well-described by the fits of Kraemer, Martin and Schulz-Blaschke equations for all three polymers except for hmHEC-2, where the plot of reduced viscosity against specific viscosity deviates from linearity at a concentration lower than $0.77 /[\eta]$.

The different levels of hydrophobic modifications within different amphiphilic hmHEC samples are also reflected in their respective values of Huggins constants given in Table 1 . The $K_{H}$ for hmHEC is 1.62 , while those of the other polymers including amphiphilic derivatives are much lower and within a close range of 0.49-0.73. Simon et al. (2003) observed that the $K_{H}$ for amphiphilic derivatives of carboxymethyl pullulans increased with increasing degree of hydrophobic modification. This trend has also been reported for hydrophobically modified derivatives of dextran (Rotureau et al. 2006), polyacrylamide (Maia et al. 2011) and carboxymethylcellulose (CharpentierValenza et al. 2005). These studies attributed the increase in $K_{H}$ to increasing polymer-polymer interaction and decreasing polymer-solvent interaction, which could also be referred to as the formation of a reversible network by supramolecular aggregation.

However, at a lower degree of hydrophobic modification such as in hmHEC-1 and hmHEC-2 of set B, the polymer backbones are able to envelope the hydrophobic groups within the coils without necessarily forming a reversible network. These polymer coils can either be made of a single polymer chain or an aggregate of more than one chain. Both intra and intermolecular hydrophobic interactions can promote such chains (Chassenieux et al. 2010). Hence the rheological behavior of such solutions slightly resembles that of a colloidal suspension of particles covered with a hydrophilic layer (Durand 2007b) below the overlap concentration $c^{*}$. Thus, the apparent thermodynamic quality of the solvent for such polymers with a low degree of hydrophobic modification is not much different from that of their non-associative derivatives. As a result the value of $K_{H}$ remains similar for associative and non-associative polymers.

The intrinsic viscosity of hmHEC is less than that of the HEC in set A. Although this is expected owing to the lower molecular mass of hmHEC compared to that of the HEC, the presence of hydrophobic associations 
also cause the collapse of the coil, making it more compact and resulting into a lower value of intrinsic viscosity. This phenomenon has been reported for the hydrophobically modified derivatives of many polymers such as dextran (Rotureau et al. 2006; Durand 2007a, b), carboxymethyl cellulose (CharpentierValenza et al. 2005), polyacrylamide (Maia et al. 2011) and hmHEC (Maestro et al. 2002a). However, for set B polymers the intrinsic viscosity values of amphiphilic derivatives are higher than that of the corresponding non-associative derivative. Considering that the molecular masses of all three polymers of set $B$ are very close, these results can be explained by the presence of supramolecular aggregates. Due to the lower degree of hydrophobic modification of hmHEC1 and hmHEC-2, the intramolecular associations are not strong enough to be able to collapse the polymer coil. Nevertheless, the presence of intermolecular hydrophobic associations results in a greater hydrodynamic volume and intrinsic viscosity for the amphiphilic polymers in set B compared to their equivalent non-associative polymer.

It must also be noted that the intrinsic viscosity of non-associative hmHEC-Blank is remarkably low compared to its precursor HEC. This can be attributed to thermal degradation of the polymer, leading to a decrease in molecular mass during the synthesis.

For each polymer within sets $\mathrm{A}$ and $\mathrm{B}$, the difference in the intrinsic viscosity values derived using Huggins, Kraemer, Martin and Schulz-Blaschke equations does not exceed $15 \%$. The $K_{H}-K_{K}$ for hmHEC (set A) is much higher than 0.5 . This has been previously reported for amphiphilic polysaccharides (Durand 2007a). Apparently due to the lower degree of hydrophobic modification, this is not the case for hmHEC-1 and hmHEC-2 (set B). In Fig. 3a, the specific viscosity of set A polymers have been plotted against their respective volume concentration $\phi$, which has been calculated as $\phi=2 / 5 c[\eta]$ (English et al. 2002), i.e. the ratio of the intrinsic viscosity of polymer coil to that of a rigid sphere (5/2) multiplied with the polymer concentration. The specific viscosity of hmHEC in set A significantly exceeds that of HEC in set A within the given range of volume concentration. This further indicates the presence of a reversible network formed by hydrophobic associations in the hmHEC (set A) solution.

For set B polymers, the specific viscosity of both amphiphilic derivatives hmHEC-1 and hmHEC-2 is only slightly greater than that of the non-associative

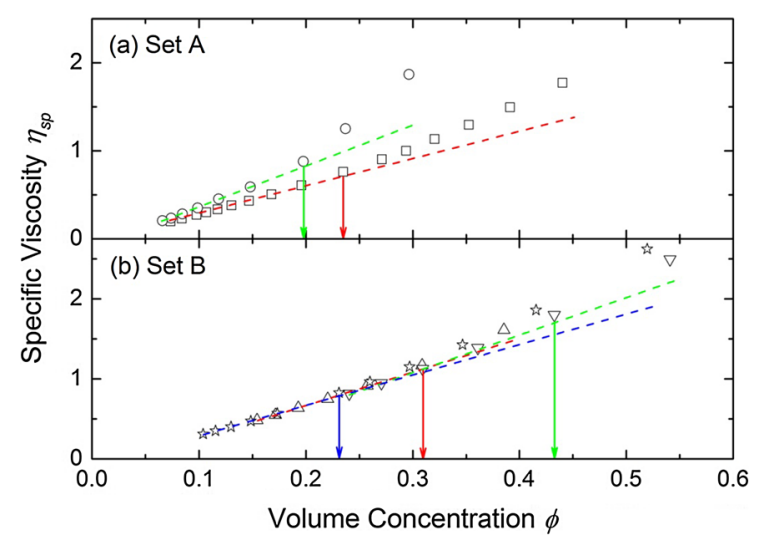

Fig. 3 a Specific Viscosity $\left(\eta_{s p}\right)$ plotted against volume concentration $\varphi=2[\eta] c / 5$ for HEC (open square) and hmHEC (open circle) in set A. Red and green broken lines are the linear extrapolations of specific viscosity values at dilute concentrations for HEC and hmHEC respectively, deviation from which is indicated by the arrows of corresponding colours. b Specific Viscosity $\left(\eta_{s p}\right)$ plotted against volume concentration $\phi$ $=2[\eta] c / 5$ for hmHEC-Blank (open triangle), hmHEC-1 (open inverted triangle) and hmHEC-2 (open star) in set B. Red, green and blue broken lines are the linear extrapolations of specific viscosity values at dilute concentrations for hmHEC-Blank, hmHEC-1 and hmHEC-2 respectively, deviation from which is indicated by the arrows of corresponding colors. (Color figure online)

hmHEC-Blank within the given range of volume concentration in Fig. 3b. This indicates the absence of a rheologically significant reversible network in hmHEC-1 and hmHEC-2 solutions at low concentrations despite the supramolecular aggregation.

The viscometric data as per the requirements of Heller and Fuoss equations for all set $\mathrm{A}$ and $\mathrm{B}$ polymers have been plotted in Fig. 4 a, b as well as Fig. 5a-c. For all five polymers, the agreement between the intrinsic viscosity values obtained from Heller and Fuoss equations is not as good compared to that between Huggins, Kraemer and Schulz-Blaschke equations. One possible explanation of this is the difference in the fundamentals of these equations. While Fuoss equation is primarily designed for polyelectrolyte solutions in which charges are not necessarily screened completely, Heller equation has been derived from combining Huggins and Kraemer equations. It is interesting that the onset of nonlinearity in Fuoss plots and Huggins plots for all the polymers occurs at very similar concentrations with an exception of hmHEC-2, where the non-linearity is observed in Heller plot instead. 


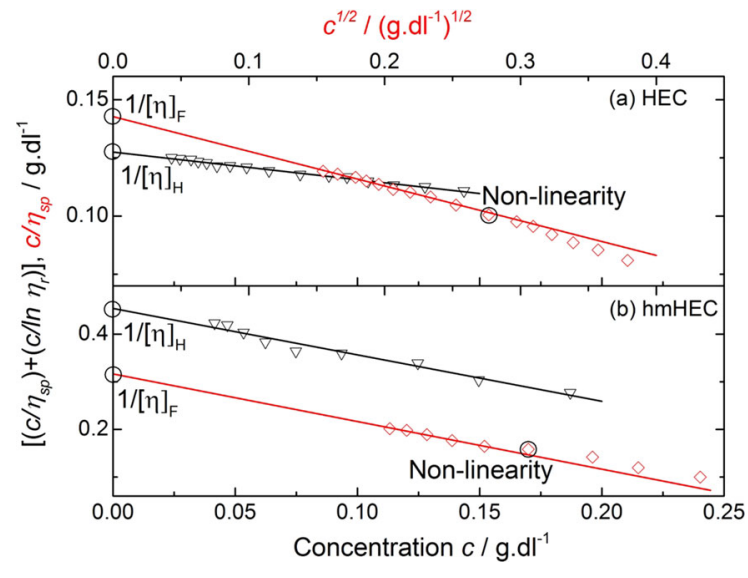

Fig. 4 The sum of the reciprocals of both reduced viscosity values $\left(c / \eta_{s p}\right)$ and inherent viscosity values $\left(c / \mathrm{ln} \eta_{\text {rel }}\right)$ plotted against concentration $c$ (open diamond) for a HEC and b hmHEC in set A. Reciprocal of reduced viscosity values $(\mathrm{cl}$ $\eta_{s p}$ ) plotted against square root of concentration $c$ (open inverted triangle) for a HEC and b hmHEC in set A. Black and red solid lines are fits for Heller and Fuoss equations respectively. The marked intercepts of these fits represent the reciprocals of intrinsic viscosity values calculated by the respective equations they represent. (Color figure online)

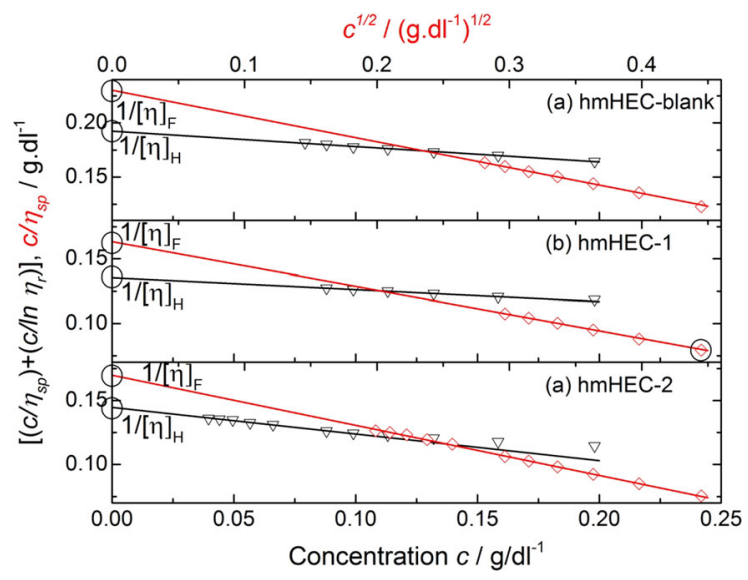

Fig. 5 The sum of the reciprocals of both reduced viscosity values $\left(c / \eta_{s p}\right)$ and inherent viscosity values $\left(c / \ln \eta_{\text {rel }}\right)$ plotted against concentration $c$ (open diamond) for a hmHEC-Blank, b hmHEC-1 and c hmHEC-2 in set B. Reciprocal of reduced viscosity values $\left(\mathrm{c} / \eta_{s p}\right)$ plotted against square root of concentration $c$ (open inverted triangle) for a hmHEC-Blank, b hmHEC-1 and c hmHEC-2 in set B. Black and red solid lines are fits for Heller and Fuoss equations respectively. The marked intercepts of these fits represent the reciprocals of intrinsic viscosity values calculated by the respective equations they represent. (Color figure online)
The viscometric data plotted in Figs. 6 and 7 is the left side term of the Fedors equation as a function of reciprocal of concentration and has been fitted with a straight line. The intrinsic viscosity values derived from Fedors plots are in a good agreement with those from the other methods with an exception of Fuoss equation. As opposed to all the previous empirical methods, these data also contain some additional points representing measurements at higher concentrations using methods other than the capillary viscometry such as rotational rheometry.

It is remarkable that Fedors equation is applicable to such a wide range of concentration for all polymers in set $\mathrm{A}$ and $\mathrm{B}$ till $c_{\text {max }}^{-1}$. For most polymers, the equation holds true even beyond the concentration defined by $c_{\text {max }}$, except for hmHEC (set A). While the Huggins plot is known to deviate from linearity beyond $c[\eta]=1$, Fedors plot maintains linearity till [ๆ] $\times c_{\text {max }}=5-9$ (Table 2) except for hmHEC (set A). The maximum values of relative viscosity covered are from $\eta_{\text {rel }}=3.87$ (hmHEC) to 534 (hmHEC-1). To the best of our knowledge, there have been no reports in the literature about any empirical relationship including Fedors equation covering $\eta_{\text {rel }}<2$ to $\eta_{\text {rel }}>500$ in one plot. We must acknowledge

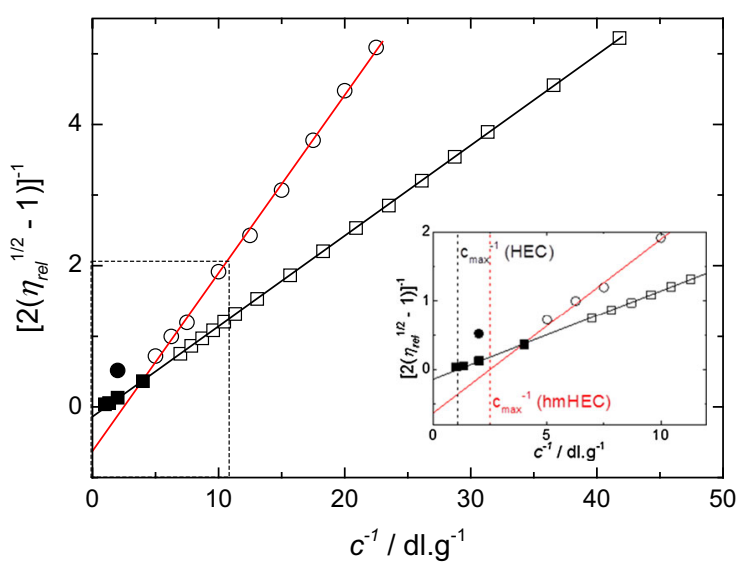

Fig. 6 The Fedors term $\left[2\left(\eta_{\text {rel }}^{1 / 2}-1\right)\right]^{-1}$ plotted against the reciprocal of concentration $c^{-1}$ for HEC (open square) and for hmHEC (open circle) within set A. Black and red solid lines are the linear fits for HEC and hmHEC, respectively. The area surrounded by the broken lines is magnified in the inset graph which represents the measurements at higher concentrations including some made with a concentric cylinder or a cone-plate geometry (solid symbols). The broken lines inside the inset graph represent the reciprocal of $c_{\max }$ obtained from Fedors equation for both the polymers within set A. (Color figure online) 


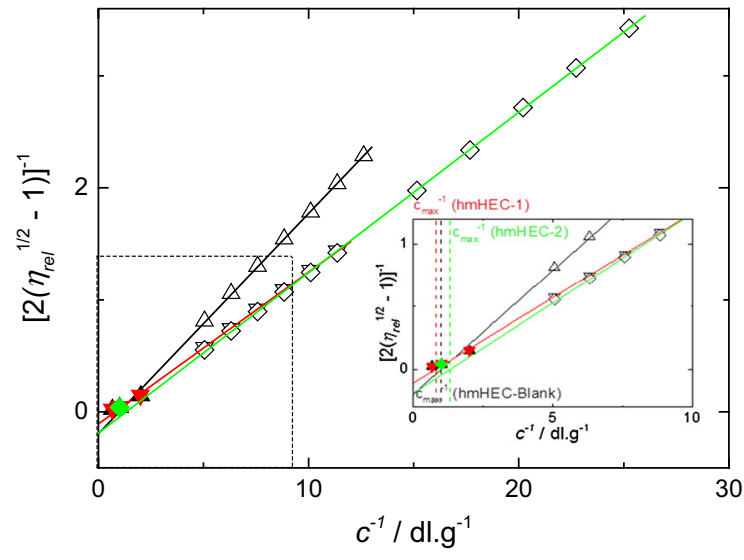

Fig. 7 The Fedors term $\left[2\left(\eta_{\text {rel }}^{1 / 2}-1\right)\right]^{-1}$ plotted against the reciprocal of concentration $c^{-1}$ for hmHEC-Blank (open triangle), for hmHEC-1 (open inverted triangle) and for hmHEC-2 (open diamond) within set B. Black, red and green solid lines are the linear fits for hmHEC-Blank, hmHEC-1 and hmHEC-2 respectively. The area surrounded by the broken lines is magnified in the inset graph which represents the measurements at higher concentrations including some made with a concentric cylinder or a cone-plate geometry (solid symbols). The broken lines inside the inset graph represent the reciprocal of $c_{\max }$ obtained from Fedors equation for all three polymers within set B. (Color figure online)

however, that at higher degree of hydrophobic modification, the upper limit for both $c_{\max }$ and $\eta_{\text {rel }}$ is substantially minimized as in the case of hmHEC (set A).

As mentioned in the theory section, Durand (2007a) established an approximate equality between

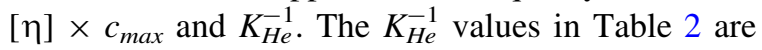
within $\pm 40 \%$ of the corresponding $[\eta] \times c_{\max }$ values for hmHEC (set A) and hmHEC-Blank (set B) and within $\pm 15 \%$ for all the other polymers.

Calculation of hydrodynamic parameters

The hydrodynamic parameters obtained for all the polymers using intrinsic viscosity measurements, static light scattering and size exclusion chromatography coupled with multi angle static light scattering and differential refractometry are listed in Table 3. Among these parameters, $M_{n}$ (number average molecular mass) and $R_{g}$ (radius of gyration) have not been measured for any associative polymers as the results are unreliable due to intermolecular aggregation. Measuring these parameters in any other (relatively less polar) solvent in which the aggregation does not take place will render the parameters incomparable with those of non-associative polymers which have been measured in an aqueous solvent. The weight average molecular mass $M_{W}$ has been taken to be the same for all polymers in set B (i.e. hmHEC-Blank, hmHEC-1 and hmHEC-2) as they have undergone the same chemical and thermal treatment.

The difference between $M_{W}, M_{n}$ and $R_{g}$ values of HEC (set A) and hmHEC-Blank (set B) confirms the intrinsic viscosity results, suggesting that thermal degradation during the synthesis has reduced the average degree of polymerization.

The data obtained from static light scattering experiments have been plotted in Fig. 8 and the value of $A_{2}$ has been calculated using the Debye equation. The second virial coefficient values $\left(A_{2}\right)$ for nonassociative polymers are higher compared to their corresponding associative polymers. This indicates lower thermodynamic solvent quality in the hmHEC solutions compared to the HEC solutions. However, the polymer-polymer interactions still do not exceed the polymer-solvent interaction as the $A_{2}$ is still positive. Such a trend has also been observed in the past among associative polyacrylamides (Berlinova et al. 2001).

The $A_{2}$ value of hmHEC-Blank is higher compared to hmHEC-1 and hmHEC-2, relating to the hydrophobic content of the latter two. Despite the difference between the degrees of hydrophobic substitution of hmHEC-1 and hmHEC-2, the $A_{2}$ values are the same,

Table 2 The intrinsic viscosity values $\left(\mathrm{dl} \mathrm{g}^{-1}\right)$ and the empirical constants obtained from Heller, Fuoss and Fedors equations for all set $\mathrm{A}$ and $\mathrm{B}$ polymers

\begin{tabular}{llllllllr}
\hline Polymer & {$[\eta]_{\mathrm{He}}$} & $K_{\mathrm{He}}$ & {$[\eta]_{\mathrm{Fu}}$} & $A$ & {$[\eta]_{\mathrm{F}}$} & $c_{\max }$ & {$[\eta]_{\mathrm{F}} \times c_{\max }$} & $K_{H e}^{-1}$ \\
\hline HEC & 7.84 & 0.119 & 6.80 & -0.170 & 7.81 & 0.923 & 7.20 & 8.44 \\
hmHEC & 2.20 & 0.974 & 3.16 & -0.555 & 3.97 & 0.403 & 1.60 & 1.03 \\
hmHEC-Blank & 5.20 & 0.142 & 4.30 & -0.245 & 5.08 & 1.01 & 5.11 & 7.03 \\
hmHEC-1 & 7.37 & 0.0926 & 6.12 & -0.188 & 7.38 & 1.26 & 9.30 & 10.8 \\
hmHEC-2 & 6.92 & 0.208 & 5.89 & -0.213 & 6.98 & 0.758 & 5.30 & 4.81 \\
\hline
\end{tabular}


Table $3 M_{W}, M_{n}, R_{g}$, second virial coefficient $A_{2}$ and other derived parameters for all polymers

\begin{tabular}{lllllll}
\hline Polymer & $M_{W} / \mathrm{g} \mathrm{mol}^{-1}$ & $M_{n} / \mathrm{g} \mathrm{mol}^{-1}$ & $R_{g} / \mathrm{nm}$ & $A_{2} / \mathrm{mol} \mathrm{dl} \mathrm{g}^{-2}$ & $A_{2} M_{W} / \mathrm{dl} \mathrm{g}^{-1}$ & $A_{2} M_{W} /[\eta]_{\mathrm{F}}$ \\
\hline HEC & $7.20 \times 10^{5}$ & $5.23 \times 10^{5}$ & 68.9 & $1.31 \times 10^{-5}$ & 9.43 & 1.21 \\
hmHEC & $3.00 \times 10^{5}$ & Not measured & & $8.95 \times 10^{-6}$ & 2.68 & 0.675 \\
hmHEC-Blank & $5.40 \times 10^{5}$ & $3.56 \times 10^{5}$ & 62.3 & $1.42 \times 10^{-5}$ & 7.66 & 1.51 \\
hmHEC-1 & $5.40 \times 10^{5}$ & Not measured & & $1.23 \times 10^{-5}$ & 6.64 & 0.900 \\
hmHEC-2 & $5.40 \times 10^{5}$ & & & $1.23 \times 10^{-5}$ & 6.64 & 0.951 \\
\hline
\end{tabular}

For hmHEC-1 and hmHEC-2, the $M_{W}$ has been taken to be the same as that of hmHEC-Blank

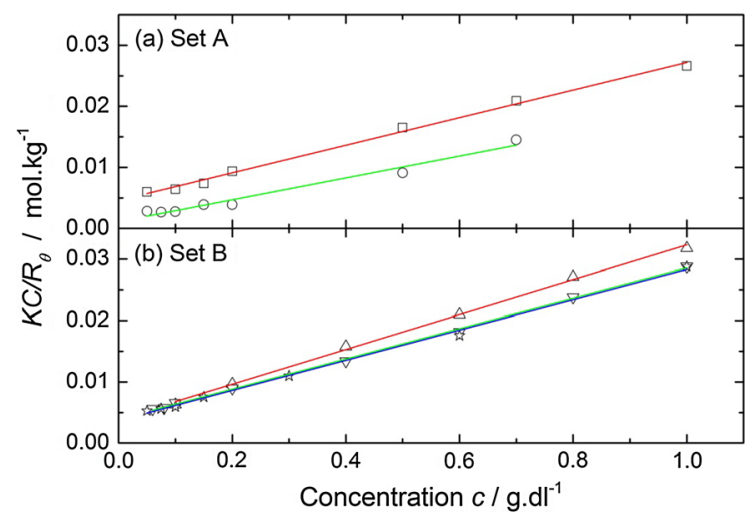

Fig. $8 K c / \mathrm{R}_{\theta}$ obtained using static light scattering has been plotted against concentration for a HEC (open square) and hmHEC (open circle) in set A and b hmHEC-Blank (open triangle), hmHEC-1 (open inverted triangle) and hmHEC-2 (open star) in set B. The data points for each polymer have been fitted with straight lines in order to calculate second virial coefficient $\left(A_{2}\right)$ values according to Debye equation. (Color figure online)

and not much lower compared to that of their corresponding non-associative polymer, possibly due to low degrees of hydrophobic modification among hmHEC-1 and hmHEC-2. This indicates that there is an absence of a reversible network between these coils and the polymer backbones are generally able to envelope the hydrophobic groups within the coils of either a single polymer chain or a supramolecular aggregate.

An approximate equality has been established between [ $\eta$ ] and $A_{2} M_{W}$. As described in Tables 2 and 3 , the $A_{2} M_{W}$ values are within $\pm 50 \%$ of their corresponding $[\eta]$ values derived from Fedors equation. The $A_{2} M_{W} /[\eta]>1.2$ for HEC (set A) and hmHEC-Blank (set B) suggest a less flexible chain in a good solvent while $A_{2} M_{W} /[\eta]<1$ for the rest of the polymers suggest the inferior solvent quality (Yamakawa 2001). However, the $A_{2} M_{W} /[\eta]$ is much lower for the hmHEC in set A compared to the amphiphilic derivatives in set $\mathrm{B}$ due to the higher degree of hydrophobic modification of the former.

\section{Conclusion}

Various empirical methods for the determination of intrinsic viscosity by extrapolation of the viscometric data were examined (i.e. Huggins, Kraemer, Martin, Schulz-Blaschke, Fuoss, Heller and Fedors) for a set of cellulose ethers with an associative and a nonassociative sample having different molecular masses and for another set of cellulose ethers with similar molecular mass but different levels of hydrophobic modification. The second virial coefficients $\left(A_{2}\right)$ for these samples were also calculated using Debye equation applied to the static light scattering data.

A reasonable agreement was observed between the values of intrinsic viscosity obtained using different equations mentioned above. The concentration parameter $c_{\max }$ was calculated using Fedors equation for each of the samples. Within the limit of this parameter, the Fedors equation successfully predicted the viscometric behavior up to $\eta_{\text {rel }} \sim 500$ even though the viscosity at such concentrations was apparently shearrate dependent. Other dimensionless empirical parameters obtained from these equations such as $K_{H}$ and $K_{H e}$ reflected the polymer-polymer and polymersolvent interactions at dilute concentrations. For hmHEC in set A, a deviation from the rheological behavior predicted by the Huggins equation was observed at a concentration lower than $0.77 /[\eta]$. This deviation and the values of $K_{H}$ and $A_{2}$ for hmHEC collectively suggested the presence of intra- and intermolecular associations and a rheologically significant network as opposed to the non-associative HEC in set A.

Within set B, the degree of hydrophobic modification was relatively lower for hmHEC-1 and hmHEC-2 
compared to the hmHEC in set A. The lower degree of hydrophobic modification enabled the hydrophilic backbone to envelope the hydrophobic groups within coils. This gave rise to intra- and intermolecular associations and supramolecular aggregates as evident by higher values of intrinsic viscosity. However, the persistently linear relationship of reduced viscosity with concentration for this polymers as well as the $K_{H}$ and $A_{2}$ values indicated that the reversible network was absent below $1 /[\eta]$ in these polymers. Thus it can be argued that even though a lower degree of hydrophobic modification is sufficient to promote intra- and intermolecular associations and aggregates, the presence of a rheologically significant reversible network is not essential.

The ratio $A_{2} M_{W} /[\eta]$ was found to be greater than 1 for both non-associative HEC polymers as a result of the semi-flexible nature of their backbones. However, for hmHEC samples sharing similar backbone, the ratio was lower due to the hydrophobic associations leading to supramolecular structures with a more compact accommodation of the chain segments within the coils.

Open Access This article is distributed under the terms of the Creative Commons Attribution 4.0 International License (http:// creativecommons.org/licenses/by/4.0/), which permits unrestricted use, distribution, and reproduction in any medium, provided you give appropriate credit to the original author(s) and the source, provide a link to the Creative Commons license, and indicate if changes were made.

\section{References}

Abdala A, Khan S, Tonelli A, Wu W, Olesen K, Jenkins R (2004) Solution rheology of hydrophobically modified associative polymers: effects of backbone composition and hydrophobe concentration. J Rheol 48(5):979-994

Berlinova I, Dimitrov I, Vladimirov N, Samichkov V, Ivanov Y (2001) Associative graft copolymers comprising a $\operatorname{poly}(N$ isopropylacrylamide) backbone and end-functionalized polyoxyethylene side chains. Synthesis and aqueous solution properties. Polymer 42(14):5963-5971

Bungenberg de Jong H, Kruyt H, Lens J (1932) Zur Kenntnis der lyophilen Kolloide XVI. Mitteilung. Viskosität und Solkonzentration. Chemische Beihefte (Fortschrittsberichte über Kolloide und Polymere) 36(11-12):429-462

Charpentier-Valenza D, Merle L, Mocanu G, Picton L, Muller G (2005) Rheological properties of hydrophobically modified carboxymethylcelluloses. Carbohyd Polym 60(1):87-94

Chassenieux C, Nicolai T, Benyahia L (2010) Rheology of associative polymer solutions. Curr Opin Colloid Sci $16(1): 18-26$
Davison G, Lane B (2003) Additives in Water-Borne coatings. The Royal Society of Chemistry, Cambridge

Dragan S, Ghimici L (2001) Viscometric behaviour of some hydrophobically modified cationic polyelectrolytes. Polymer 42(7):2887-2891

Dumitriu S (2001) Polymeric biomaterials, 2nd edn. Marcel Dekker Inc, New York

Dumitriu S (2005) Polysaccharides: structural diversity and functional versatility, 2nd edn. Marcel Dekker, Quebec

Durand A (2007a) Aqueous solutions of amphiphilic polysaccharides: concentration and temperature effect on viscosity. Eur Polym J 43(5):1744-1753

Durand A (2007b) Semiempirical equations for the viscosity of amphiphilic polymer solutions: a critical examinations. Polym Eng Sci 47(4):481-488

Einstein A (1920) Bemerkung zu der Abhandlung von W. R. Heß „Beitrag zur Theorie der Viskosität heterogener Systeme“. Colloid Polym Sci (Kolloidzeitschrift in 1920) 27(3): 137

English R, Laurer J, Spontak R, Khan S (2002) Hydrophobically modified associative polymer solutions: rheology and microstructure in the presence of nonionic surfactants. Ind Eng Chem Res 41(25):6425-6435

Fedors R (1979) An equation suitable for describing the viscosity of dilute to moderately concentrated polymer solutions. Polymer 20(2):225-228

Fuoss R, Strauss U (1948) Polyelectrolytes. II. Poly-4vinylpyridonium Chloride and Poly-4-vinyl-N-nbutylpyridonium Bromide. J Polym Sci 3(2):246-263

Gonzalez J, Muller A, Torres M, Saez A (2005) The role of shear and elongation in the flow of solutions of semi-flexible polymers through porous media. Rheol Acta 44:396-405

Goodwin J, Hughes R (2008) Rheology for chemists: an introduction, 2nd edn. The Royal Society of Chemistry, Cambridge

Graessley W (1980) Polymer chain dimensions and the dependence of viscoelastic properties on the concentration, molecular weight and solvent power. Polymer 21:258-262

Heller W (1954) Treatment of viscosity data on polymer solutions (An Analysis of Equations and Procedures) I. Intrinsic viscosity and limiting slope constant. J Coll Sci 9(6):547-573

Huggins M (1942) The viscosity of dilute solutions of longchain molecules. IV. Dependence on concentration. J Am Chem Soc 64(11):2716-2718

Islam M, Jenkins R, Bassett D, Lau W, Yang H (2000) Single chain characterization of hydrophobically modified polyelectrolytes using cyclodextrin/hydrophobe complexes. Macromolecules 33(7):2480-2485

Kamide K, Dobashi T (2000) Physical chemistry of polymer solutions, 1st edn. Elsevier, Amsterdam

Klemm D, Philipp B, Heinze T, Heinze U, Wagenknecht W (1998) Comprehensive cellulose chemistry volume 2: functionalization of cellulose. Wiley-VCH, Germany

Kraemer E (1938) Molecular weights of celluloses and cellulose derivates. Ind Eng Chem 30(10):1200-1203

Kulicke W, Clasen C (2004) Viscosimetry of polymers and polyelectrolytes. Springer, Hamburg

Landoll L (1982) Nonionic polymer surfactants. J Polym Sci Polymer Chem Edition 20:443-455 
Laschet M, Plog J, Clasen C, Kulicke W (2004) Examination of the flow behaviour of HEC and hmHEC solutions using structure-property relationships and rheo-optical methods. Colloid Polym Sci 282(4):373-380

Lau A, Tiu C, Kealy T, Tam K (2002) Rheology of hydrophobic-alkali-soluble emulsions and the effect of surfactants. Korea-Aust Rheol J 14(1):1-9

Lee D (1992) Molecular dimension of poly(organophosphazene): 2 Unpurturbed molecular dimension of poly[bis(phenylphenoxy)-phosphazene]. Polymer (Korea) 16(3):351-362

Ma J, Liang B, Cui P, Dai H, Huang R (2003) Dilute solution properties of hydrophobically associating polyacrylamide: fitted by different equations. Polymer 44(4):1281-1286

Maestro A, Gonzalez C, Gutierrez J (2002a) Rheological behaviour of hydrophobically modified hydroxyethyl cellulose solutions: a linear viscoelastic model. J Rheol 46:127-143

Maestro A, Gonzalez C, Gutierrez J (2002b) Shear thinning and thixotropy of HMHEC and HEC water solutions. J Rheol 46(6): 1445-1457

Maia A, Villetti M, Vidal R, Borsali R, Balaban R (2011) Solution properties of a hydrophobically associating polyacrylamide and its polyelectrolyte derivatives determined by light scattering, small angle $\mathrm{X}$-ray scattering and viscometry. J Braz Chem Soc 22(3):489-500

Martinez-Richa A (2012) Determination of molecular size of O-(2-hydroxyethyl) cellulose (HEC) and its relationship to the mechanism of enzymatic hydrolysis by cellulases. Carbohyd Polym 87(3):2129-2136

Martins R, da Silva C, Becker C, Samios D, Christoff M, Bica C (2006) Interaction of (hydroxypropyl) cellulose with anionic surfactants in dilute regime. Colloid Polym Sci 284(12):1353-1361

Patruyo L, Muller A, Saez A (2002) Shear and extensional rheology of solutions of modified hydroxyethyl celluloses and sodium dodecyl sulfate. Polymer 43:6481-6493
Pavlov G, Gubarev A, Zaitseva I, Sibileva M (2006) Determination of intrinsic viscosity of polyelectrolytes in salt-free solutions. Physicochem Stud Syst Process 79(9):14071412

Podzimek S (2011) Light Scattering, size exclusion chromatography and asymmetric flow field flow fractionation, 1st edn. Wiley, New Jersey

Rotureau E, Dellacherie E, Durand A (2006) Viscosity of aqueous solutions of polysaccharides and hydrophobically modified polysaccharides: application of fedors equation. Eur Polym J 42(5):1086-1092

Rubinstein M, Dobrynin A (1997) Solutions of associative polymers. Trends Polym Sci 5(6):181-186

Schulz G, Blaschke F (1941) Eine Gleichung zur Berechnung der Viscositatszahl fur sehr kleine Konzentrationen. J Prakt Chem 158(1):130-135

Simon S, Dugast J, Cerf D, Picton L, Muller G (2003) Amphiphilic polysaccharides. evidence for a competition between intra and intermolecular associations in dilute system. Polymer 44(26):7917-7924

Swift G, Carraher C, Bowman C (1997) Polymer modification. Plenum Press, New York

Teraoka I (2002) Polymer solutions. Wiley, New York

van't Hoff J (1903) Vorlesungen uber Theoretische und Physikalische Chem, 1st edn. F. Vieweg und Sohn, Braunschweig

Whistler R, BeMiller J (1993) Industrial gums: polysaccharides and their derivatives, 3rd edn. Academic Press Limited, London

Yamakawa H (2001) Modern theory of polymer solutions, Electronic edn. Harper \& Row, Kyoto

Zhang L (2001) Cellulosic associative thickeners. Carbohyd Polym 45(1):1-10

Zhao G, Chen S (2007) Nonlinear rheology of aqueous solutions of hydrophobically modified hydroxyethyl cellulose with nonionic surfactant. J Colloid Interf Sci 316(2):858-866 